\title{
Clostridium difficile Associated Disease Clinical and Molecular Data
}

\author{
Karina Aksenoka*,**, Arta Balode ${ }^{* *, * * *}$, Ilze Grope****, Tatjana Obidenova ${ }^{* * *}$, Dace Gardovska ${ }^{* *}$, ${ }^{* * * *}$, Edvins \\ Miklasevics*,** \\ *Department of Molecular Biology and Genetics, Pauls Stradins Clinical University Hospital, Riga, Latvia \\ **Riga Stradins University, Latvia \\ ***Department of Microbiology, Pauls Stradins Clinical University Hospital, Riga, Latvia \\ **** Children`s University Hospital, Riga, Latvia
}

\begin{abstract}
Summary
Introduction. A balanced microflora of the gastrointestinal tract plays a significant role in the protection of an organism. Dramatic changes may take place in the composition of normal microflora during antibacterial therapy, because part of sensitive microorganisms die and microflora detrimental to an organism proliferates there causing gastrointestinal tract disorders (7).

C. difficile is an important agent causing gastrointestinal tract disorders. It may induce manifestations ranging from asymptomatic colonization of the gastrointestinal tract to severe diarrhea, pseudomembranous colitis, toxic megacolon, intestinal perforation, and death. All these severity levels are included in the term Clostridium difficile associated disease (CDAD). A patient at risk for $C$. difficile is an elderly person with a severe principal disease, who receives long-term inpatient treatment, has been receiving antibiotics, antacid and antiulcer agents for a long time, has had a gastrointestinal surgical intervention or any organ transplantation, which is always related to immune suppression (11).

Aim of the study. Molecular typing of Latvian strains of $C$. difficile and clinical data analysis.

Materials and methods. The research includes data collected during the time period from August 2006 to the end of 2008 from three Latvian hospitals. A pure culture of $C$. difficile bacteria was isolated and cultured from fecal material. Ribotyping was done by amplification of specific regions of the 16S and 23S RNA genes and amplification products were separated in 1.5\% agarose gel.

Results. Fecal samples of 500 patients having manifestations of clinical symptoms of CDAD were microbiologically and molecularly studied from August 2006 to the end of 2008. 130 samples were $t c d A^{+} t c d B^{+}$toxin-positive.

Conclusions. All samples were cultured on agar in anaerobic conditions. As a result, 55 pure cultures were obtained. The average age of patients is 65 years. Women get infected more frequently than men. In 95\% patients CDAD was induced by antibacterial therapy. In $86 \%$ cases, having received a positive response for C.difficile antibacterial therapy is changed. Disease development risk factors are previous antibacterial therapy, previous hospitalization, GIT manipulations and C.difficile infection in anamnesis. The biggest number of CDAD patients was found in Nephrology Department of P.Stradins Clinical University Hospital. A hypervirulent Cl.difficile ribotype 027 was not found in Latvia yet, bat 16 different ribotypes were determined using Bionumerics software. 4 of them are domineering ribotypes with 17, 14, 5 and 4 isolates in every group.
\end{abstract}

Key words: C.difficile; $C D A D ;$ tcdA and tcdB.

\section{INTRODUCTION}

A balanced microflora of the gastrointestinal tract plays a significant role in the protection of an organism. Dramatic changes may take place in the composition of normal microflora during antibacterial therapy, because part of sensitive microorganisms die and microflora detrimental to an organism proliferates there causing gastrointestinal tract disorders. The most widespread endogenous infection related to use of antibiotics is candida, induced by fungi of the genus Candida and Clostridium difficile (7).

C. difficile is an important agent causing gastrointestinal tract disorders. It may induce manifestations ranging from asymptomatic colonization of the gastrointestinal tract to severe diarrhea, pseudomembranous colitis, toxic megacolon, intestinal perforation, and death. All these severity levels are included in the term Clostridium difficile associated disease (CDAD). A patient at risk for $C$. difficile is an elderly person with a severe principal disease, who receives long-term inpatient treatment, has been receiving antibiotics, antacid and antiulcer agents for a long time, has had a gastrointestinal surgical intervention or any organ transplantation, which is always related to immune suppression (11).

The increase in C.difficile infection rates for the last three decades may be explained by:

1. Improvement of diagnostic techniques;

2. Widespread use of antibiotics and chemotherapy;

3. Contamination of hospitals with C.difficile spores (9).

C. difficile is a gram-positive, spore-forming, anaerobic, rod-shaped bacterium of the genus Bacillus. It may be found in many objects, therefore it is called a ubiquitous (being everywhere) microorganism. It is present in soil (up to $21 \%$ ), water, intestinal tract of many animals. It is also present, though not very often, in the intestinal tract of healthy people $-C$. difficile may be isolated from feces of not more than $5 \%$ of healthy people; C.difficile is 
present in feces of up to $50 \%$ of healthy newborns, but it does not cause disease, because babies are protected by antibodies received from their mothers. Sometimes the microorganism may be also found in the vagina and in the urethra(14). The colonization percentage among elderly people, especially those who received inpatient treatment, may reach $10-20 \%$, because the hospital environment is often contaminated with $C$. difficile spores. Vegetative bacteria die, but spores may live for months and even years (20).

Colonizing the large intestine C.difficile generates toxins. C. difficile generates two toxins - $\mathrm{A}(t c d A)$ and $\mathrm{B}(t c d B)$. Toxin production is determined by a chromosomal gene. Toxin production and microorganism's virulence depends on the degree of gene expression (6).

Less than $1 \%$ are symptom-free carriers of C.difficile. Factors of host and microorganism determining symptom-free carriage are not clear. Symptoms appear either during use of antibiotics or shortly after it. In rare cases the interval may be several weeks. Patients most frequently have mild or moderately severe diarrhea, they may also have loose stool with occult blood. Rarely, it may be accompanied by pains in the lower belly. Severe colitis, without formation of pseudomembranes, characterized by profuse, severe diarrhea, stomach aches, and abdominal swelling, may develop. The most frequent systematic symptoms are sickness, fever, loss of appetite, tiredness. Rarely, hemorrhagic colitis develops. Diffuse or fragmentary nonspecific colitis is visible on the sigmoidoscopy. Severe colitis with formation of pseudomembranes is characterized by pronounced severe diarrhea, stomach aches. Characteristic yellow pseudomembranes, 2 to $10 \mathrm{~mm}$ in diameter, are visible on the sigmoidoscopy, the mucous membrane between them looks healthy or slightly eritematous. An acute stomach pattern with pronounced stomach aches, fever, and tachycardia may develop in patients. As a result of loss of muscular tone toxic megacolon may develop, which is a state usually characteristic of ulcerative colitis (16).

When paralytic ileus and large intestine dilatation develop, a pathologic decrease in the volume of diarrhea is observed. If stomach aches increase, intestinal perforation and peritonitis should be excluded. Anyway, disease symptoms may be different and may change every several hours, therefore it is important to follow up the patient and to evaluate the situation in an adequate way (5).

Since 2002 special attention has been attracted to a hypervirulent strain of $C$. difficile which belongs to toxinotype III (ribotype 027) and initially spread in the USA and Canada $(18,19)$, but now has already caused hospital outbreaks in Ireland (17), Belgium (8), France (23), Denmark (22), Austria (10) and other countries $(4,13)$.

Taking into consideration that a very pathogenic $C$. difficile ribotype 027 causing very serious disease is currently spreading in the USA and Europe, the research of this microbe becomes very topical in Latvia as well in order to perform not only diagnostics, but also ribotyping of this microorganism to start adequate therapy in case of necessity and perform infection control measures.

All CDAD patients need patient-centered approach, developing the most optimal and effective treatment regimes. Since $\mathrm{CDAD}$ is a severe infectious disease requesting the use of many different drugs (PDS, antibacterial, cardiological agents, anaesthetics, and resolvents, etc.), the presence of a hospital pharmacist could significantly optimize patient's treatment as well as reduce treatment costs . It is important to carry out a separate research on the situation in Latvia, because in case of minor microbiological changes in the circulating inducer, the process and the course of treatment may differ from the experience of other countries.

\section{AIM OF THE STUDY}

Molecular typing of Latvian strains of $\mathrm{Cl}$. difficile and clinical data analysis.

\section{MATERIALS AND METHODS}

The research includes data collected during the time period from August 2006 to the end of 2008 from three Latvian hospitals - P. Stradins Clinical University Hospital,

Gailezers hospital and Traumatology and Orthopedic Hospital. A questionnaire applied only to patients of $\mathrm{P}$. Stradins Clinical University Hospital and a retrospective study was performed within the framework of this research.

Bacterial isolates. DNA was isolated from feces which were sent to the laboratory being suspect to CDAD, using a kit QIAamp DNA Stool mini Kit (Qiagen, Germany) and it was determined whether they contained toxin genes $t c d A$ and $t c d B$ by PCR (16). A pure culture of $\mathrm{Cl}$. difficile bacteria was isolated and cultured from fecal material. Each material was cultured on $\mathrm{Cl}$. difficile selective agar (Clostridium difficile Selective Agar, Becton Dickinson, USA), blood agar and CNA blood agar, according to anaerobic culture growth methods (20). On blood agar and CNA blood agar Cl. difficile is of medium-size, forming colonies with a concentrated whitish center and a transparent, wavy area around it. Colonies are characterized by 'smell of a horse'. On Cl. difficile selective agar colonies grow in a similar way, but the colour of colonies change as a result of fission of fructose (mannose).

Ribotyping. Ribotyping was done by amplification of specific regions of the $16 \mathrm{~S}$ and 23S RNA genes and amplification products were separated in $1.5 \%$ agarose gel $(12,15)$. The filogenetic analysis of ribotypes was performed using BioNumerics (Applied Maths BVBA) software (1).

\section{RESULTS}

Fecal samples of 500 patients having manifestations of clinical symptoms of CDAD were microbiologically and molecularly studied from August 2006 to the end of 2008. 130 samples were $t c d A^{+} t c d B^{+}$toxin-positive. All samples were cultured on agar in anaerobic conditions. 
As a result, 55 pure cultures were obtained. All cultures comprised toxinogenous Cl.difficile bacteria, which were ribotyped, i.e. the arrangement of ribosomal genes in a specific strain was determined (Figure 1).

As a result of filogenetic analysis 16 different ribotypes were determined using Bionumerics software. 4 of them are domineering ribotypes with 17, 14, 5 and 4 isolates in every group. (Figure 2 ).

Having analyzed data from appointment card forms, it can be concluded that the average age of patients is $65(+/-17.12)$ years. Having distributed patients by age groups with a 5 year interval it is seen that mainly people older than 60 get infected. (Figure 3).

Distribution by gender: 35 women $(64 \%)$ and 20 men $(36 \%)$. Almost all patients, i.e.

52 patients $(95 \%)$, received antibacterial therapy, 3 patients $(5 \%)$ did not receive it. All patients had serious clinical diagnoses and were treated for a long time both at home and in hospital.

21 patient of all 55 patients were from P.Stradins Clinical University Hospital. The analysis by departments clearly demonstrates that the biggest number of confirmed Clostridium difficile patients is in the Nephrology Department (Figure 4). The development of clinical presentation of CDAD was similar in all patient, diarrhea and fever were prevailing (Figure 5). Retrospectively analyzing possible risk factors for CDAD development, it was traced that the majority of patients were prescribed antibacterial therapy in hospital (18 patients), 10 patients had already been hospitalized during previous two months, 8 patients were performed different GIT manipulations and 3 patients had C.difficile infection in their anamnesis (Figure 6). Having received a positive response for $C$.difficile antibacterial therapy is changed in $86 \%$ cases, it is changed to metronidazole or vancomycin (Figure 7).

\section{DISCUSSION}

Analysis of the clinical data was performed during the research for patients having laboratory-proved $C$. difficile infection. The average age of patients of the study is 65 years. CDAD patients are elderly people also according to literary data from all over the world (2). In 95\% of patients the infection was induced by antibacterial therapy used for treatment of the principal disease. Having received a positive response for $C$.difficile positive toxin, the therapy was changed to metronidazole or vancomycin - agents selected for treatment of C.difficile infection - in $86 \%$ cases. The infection may develop in 5 to $30 \%$ of patients receiving antibacterial therapy (3). Due to the fact that the range of antibiotics becomes wider, the incidence of the disease has increased over the past years. Currently, C.difficile is resistant to all antibiotics, except vancomycin and metronidazole, scientists, however, consider that new bacterial strains may become resistant to these antibiotics as well. Thus antibacterial agents are the main cause of outbreaks of iatrogenic diarrhea, and it significantly increases mortality and inpatient treatment costs (2).

$20 \%$ of hospitalized patients get infected by C.difficile infection in hospital and diarrhea develops in 30\% of them (5). The development of $C$. difficile bacterium is also stimulated by patient's complicated severe principal diagnosis making the patient to receive long-term inpatient treatment and have diverse examinations related to it in hospital, in particular gastrointestinal tract examinations. It is proved by my research data.

A toxicogenic ribotype 027 is not spread in Latvia yet, but 16 other different ribotype strains inducing severe health impairments are spread. British scientists have already proved that according to their studies $C$. difficile is able to collect mutations and exchange genes incredibly quickly, in this way ensuring its viability. $10 \%$ of the genome consist of mobile phases, i.e. the nucleotide sequence may migrate from one organism to the other, as a result a bacterium with genes making it so pathogenic appears (21). Therefore, the relation of other ribotypes to clinical presentations should be evaluated and infection control measures should be implemented. The control possibilities of this infection are not complex, but are labour-consuming. The use of high-risk antibacterial agents should be reduced and the spread of $C$. difficile spores in hospitals should be restricted. Experimental data demonstrate that in the ward, in which an infected patient was accommodated, one third of environment samples contain spores of the bacterium, but in control wards only $1.3 \%$ of samples are positive (13). Therefore, hygiene plays a very important role in the restriction of spread of the infection.

\section{CONCLUSIONS}

1. The average age of patients is 65 years.

2. Women get infected more frequently than men, distribution by gender: 35 women $(64 \%)$ and 20 men $(36 \%)$.

3. In $95 \%$ patients CDAD was induced by antibacterial therapy.

4. In $86 \%$ cases, having received a positive response for C.difficile antibacterial therapy is changed.

5. Disease development risk factors are previous antibacterial therapy, previous hospitalization, GIT manipulations and C.difficile infection in anamnesis.

6. The biggest number of CDAD patients was found in Department of Nephrology in Pauls Stradins Clinical University Hospital.

7. A hypervirulent Cl.difficile ribotype 027 was not found in Latvia yet, bat 16 different ribotypes were determined using Bionumerics software. 4 of them are domineering ribotypes with 17, 14, 5 and 4 isolates in every group.

\section{Conflict of interest: None}

\section{REFERENCES}

1. Bidet $\mathrm{P}$, Lalande V, Salauze B, et al. Comparison of PCR - Ribotyping, Arbitrarily PCR and Pulse - Field Gel Electrophoresis for Typing Clostridium difficile // J Clin Microbiol, 2000; 38:2484 - 2487 
2. Biller P, Shank B, Lind L, et al. Moxifloxacin Therapy as a Risk Factor for Clostridium difficile Associated Disease During an Outbreak:Attempts to Control a New Epidemic Strain // Infect Control Hosp Epidemiol, 2007; 28:199 - 201

3. Bouza E, Burillo A, Munoz P. Antimicrobial Therapy of Clostridium difficile-Associated Diarrhea // Med Clin North America, 2006; 90:1141 - 1163

4. Chandler R.E, Hedberg K, Cieslak R. Clostridium difficile-Associated Disease in Oregon: Increasing Incidence and Hospital-level Risk factor // Infect Control Hosp Epidemiol, 2007; 28:1 16 - 122

5. Clifford L, Coignard B. Recommendations for Surveilance of Clostridium difficile - Associated Disease // Infect Control Hosp Epidemiol, 2007; 28:140-145

6. Coignard B, Barbut F, Blanckaert K, et al. Emergence of Clostridium difficile toxinotype III, PCR-ribotype 027 - associated disease // Euro Surveill, 2006; 11: pii $=060914$

7. Daniel E., Ballard D. Clostridium difficile Toxins: Mechanism of Action and Role in Disease // CMR, 2005; 18:247-263

8. Delme M, Ramboer I, Van Broeck I, et al. Epidemiology of Clostridium difficile toxinotype III, PCR-ribotype 027 associated disease in Belgium // Euro Surveill, 2006; 1 1:3045

9. Gerding N, Johnson S., Peterson R, et al. Clostridium difficile - associated diarrhea and colitis // Infect Control Hosp Epidemiol, 1995; 16:459 - 477

10. Indra A, Huhulescu S, Hasenberger $\mathrm{P}$, et al. First isolation of Cl. difficile PCR ribotype 027 in Austria // Euro Surveill, 2006; 11 :pii=3046

11. Kelly P, Pothoulakis C, LaMont T. Clostridium difficile colitis // NEJM, 1991; 330:257 - 262

12. Killgore G, Thompson A, Johnson S, et al. Comparision of Seven Technigues for Typing International Epidemic Strains of Clostridium difficile:REA, PFGE, PCR-ribotyping, MLST, MLVA, AFLP, and slpAST // J Clin Microbiol, 2008; 46: $432-437$

13. Kuijper J, Coignard B, Tüll P. Emergence of Clostridium difficile-associated disease in North America and Europe // CMI, 2006; 12:2-18

14. KuijperJ, SurawiczM.Clostridium difficileinfection// Lancet, 2008; 371:1486 - 1488
15. Lemee L, Bourgeois I, Ruffin E, et al. Multilocus sequence analysis and comparative evolution of virulence-associated genes and housekiping genes of Clostridium difficile // J Clin Microbiol, 2005; $46: 3171-3180$

16. Lemee L, Dhalluin A, Pestel-Caron $M$, et al. Multilocus sequence typing analysis of human and animal Clostridium difficile isolates of various toxigenic types // J Clin Microbiol, 2004; 28: $2609-1617$

17. Long S, Fenebon L, Fitzgerald S, et al. First isolation and report of cluusters of C.difficile PCR 027 cases in Ireland // Euro Surveill, 2007; 12:pii=3183

18. Pepin J, Valquette L, Alary E, et al. Clostridium difficile-Associated diarrhea in a region of Quebec from 1991 to 2003: a cahgning pattern of disease severity // CMAJ, 2004; 171:466-472

19. Pepin J, Valiguette L, Cossete B. Mortality attributall to nosocomial Clostridium difficile-associated disease during an epidemic caused by a hypervirulent strain in Quebec // CMAJ, 2005; 170:1037-1042

20. Poxton R, McCouberey J, Blair G. The pathogenicity of Clostridium difficile // Clin Microbiol Infect, 2001; 7:421-427

21. Rupnik M, Aveseni V, Janc M., et al. Toxinotyping Schema and Correlation of Toxinotypes with Serogroups of Clostridium difficile Isolates // J Clin Microbiol, 1998; 23:2240 - 2247

22. Soes L, Mollak K, Trobek S,et al. The emergence of Cl. difficile PCR Ribotype 027 in Denmark // Euro Surveill, 2009; 14:19183

23. Tachon M, Cattone C, K.Balanckaert, et al. First cluster of Clostridium.difficile toxinotype III, PCRribotype 27 associated disease in France: prelimunary report // Euro Surveill, 2006; 11:2951

\section{Address:}

Karina Aksenoka

Department of Molecular Biology and Genetics

Pauls Stradins Clinical University Hospital

Pilsonu Street 13,

Riga, Latvia, LV-1003

e-mail: karina.a@inbox.lv 


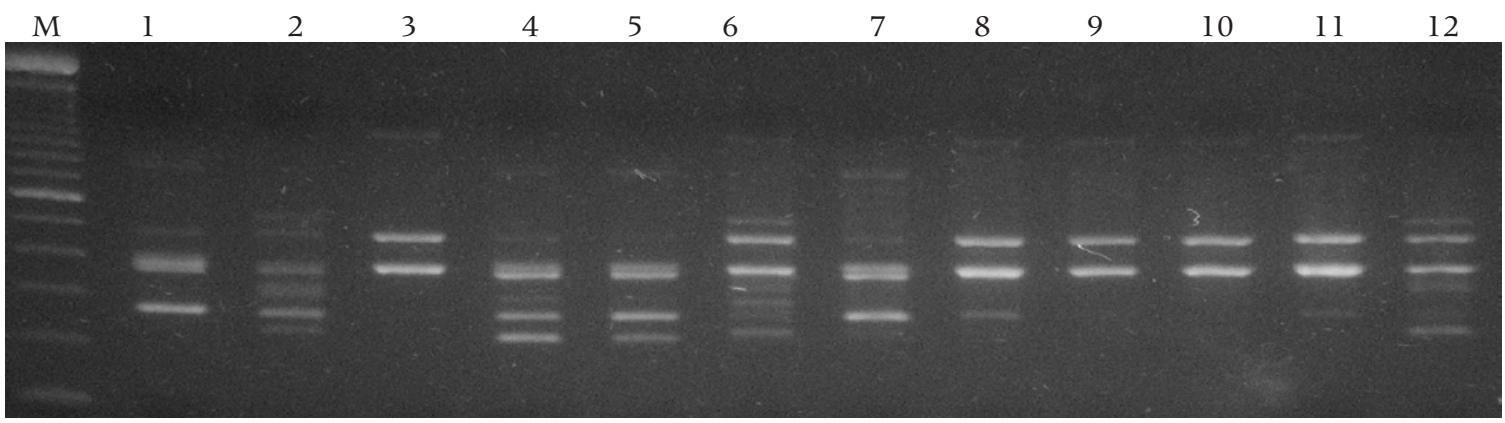

Fig. 1. Separation of ribotyping amplification products in $3 \%$ agaroze gel. $1-12$ different isolates. $M-$ DNA marker (100bp DNA Ladder, Invitrogen)

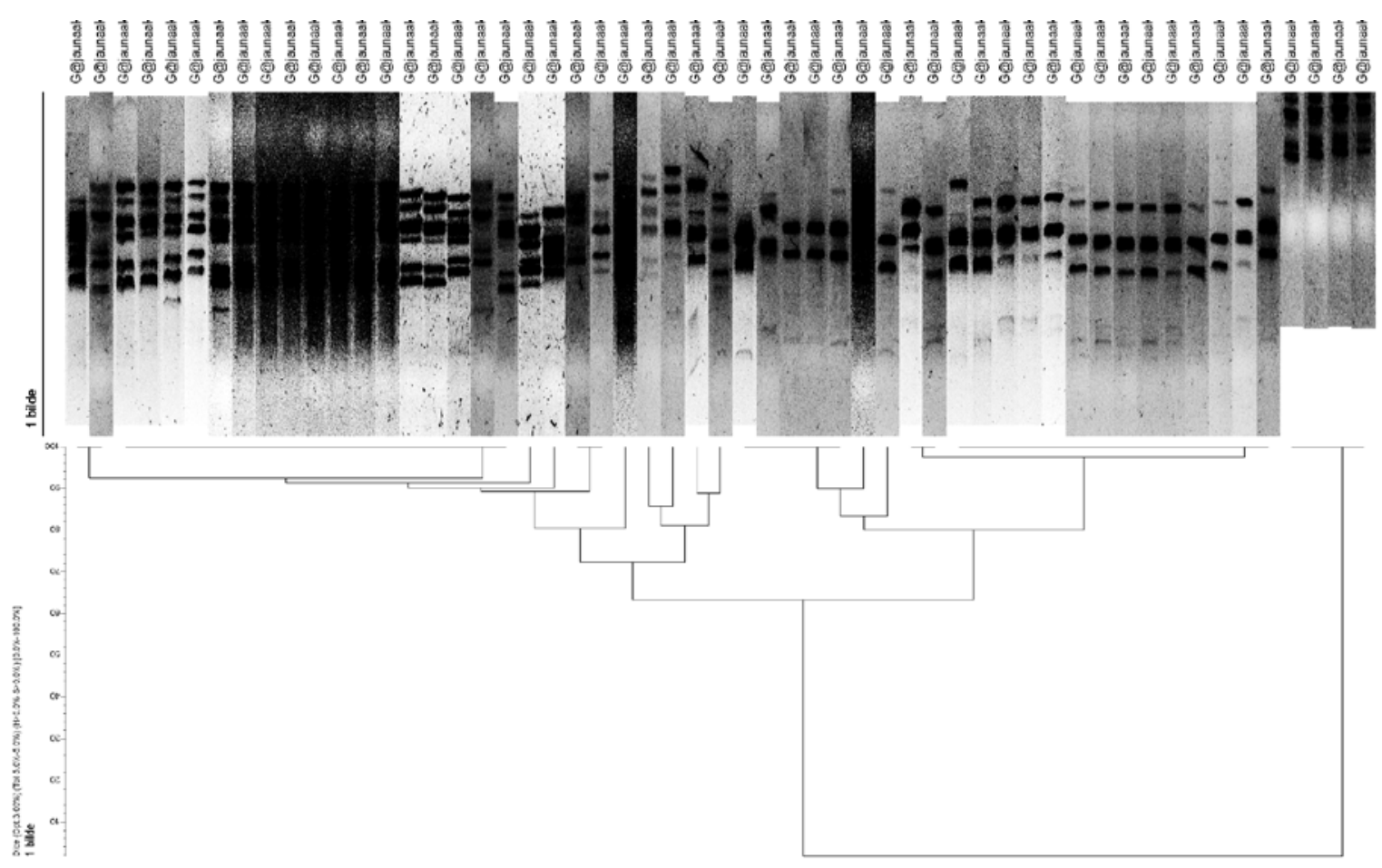

Fig. 2. Filogenetic tree of Cl.difficile isolates 


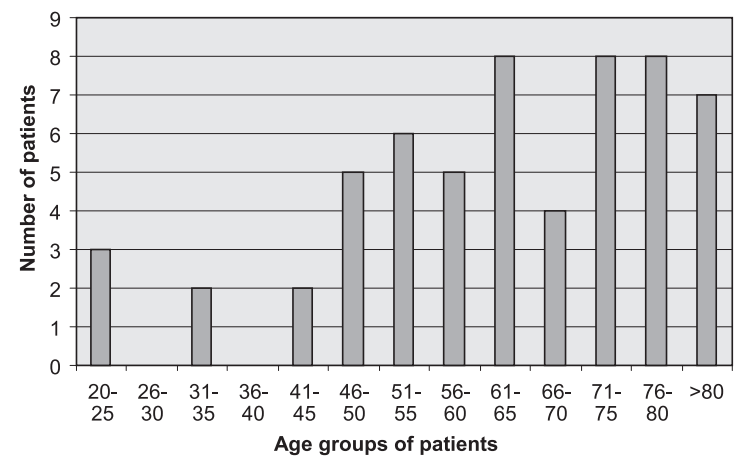

Fig. 3. Age distribution of patients with Clostridium difficile

Percentage of C.difficile in each Department

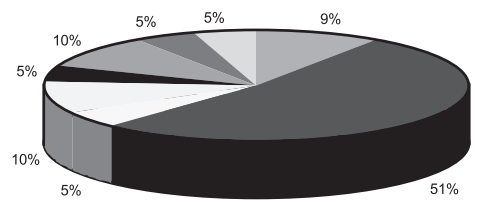

$$
\begin{aligned}
& \text { - Nephrology } \\
& \square \text { Thoracic surgery } \\
& \square \text { Surgery } \\
& \text { - Endocrinology } \\
& \square \text { Cardiology } \\
& \square \text { solator } \\
& \square \text { Neurosurgery }
\end{aligned}
$$

$\square$ Gastroenterology

Fig. 4. The percentage of Clostridium difficile patients in each department in Pauls Stradins Clinical Unversity Hospital

\section{Clinical presentation of C.difficile}

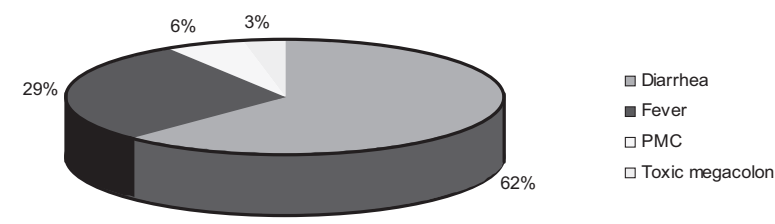

Fig. 5. Clinical presentation of C.difficile infection

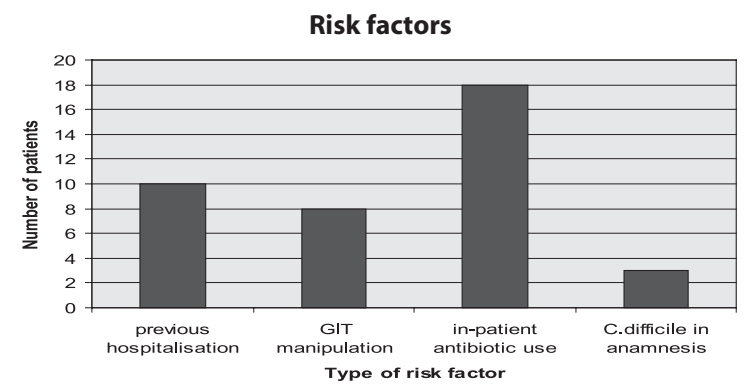

Fig. 6. Risk factors for the development of Clostridium difficile infection

\section{Changes in antibiotic}

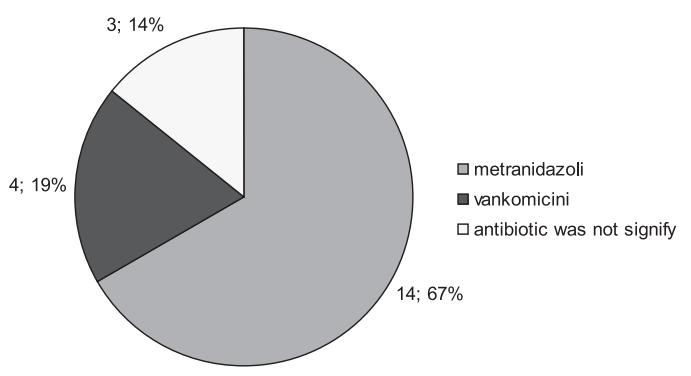

Fig. 7. Changes made to the administration of high risk antibiotics that have been implicated as risk factors for development of Clostridium difficile 